\title{
INDEX TO ABANDONED TOIVNS OF IOWA
}

\author{
By David C. Mo'TT
}

There has appeared in each of the five preceding numbers of the ANNals an installment of our article on abandoned towns, villages and post offices of Iowa. The subject was treated by taking one county at a time and arranging the counties alphabetically. A brief amount of information about each place was given as to location, date of existence, and as to whether it was a town, village, hamlet or only a country post office, when that information could be obtained.

In our introductory article, Vol. XVII, No. 6, p. 435, we set out our plan and gave our principal sources of information. $\Lambda$ s we then said "we only claim for this survey such accuracy as was made possible by the authorities at hand." In many counties, however, we received very fine help from persons who thoroughly knew their counties, while in some other counties we failed to get the careful co-operation which we desired.

Having treated the places by counties and with some information about each place as above stated, we are appending below all of the 2,807 names in alphabetical order, giving no information except the name of the county. This list will enable one to quickly find the names of a great many old towns or villages or country post offices that have long since passed away.

for several reasons these 2,807 names do not mean that that cntire number of places-towns, villages, or country post offices -are abandoned.

Some towns or villages have been absorbed by adjacent cities or towns, and have become a part of the larger corporations, losing their former identities, and finally their names. There were 46 of these. Witness Boonesboro now a part of Boone, Lyons now a part of Clinton, Sevastopol now a part of Des Moines.

Some have only lost their original names and assumed others, such as Bloomington, now Muscatine; Montana, now Boonc; Grant, now Clarion. Some even lost more than one original name, such as Miller's Hollow, changed to Kanesville, and finally 
to Council Bluffs. In all there were in our list of 2,807 names, 362 of them that are only names discarded for other names.

Some of the abandoned towns really bore two names, the name of the post office being different from the name of the town. I.ithopolis was the name of the post office at Steamboat Rock, Hardin County, for many years. Amazon was the name of the post office at the village of Richmond, Madison County, and so on. Then some of the small. towns had nicknames, or double names, such as Harpersville, or Stringtown, Davis County. There were 119 of these old places with double names by reason of having nicknames, or of having post offices with names differing from the names of their towns.

There were 75 towns laid out, or staked off, and named, most of them getting on the county records and on the maps of those days, that were never built up. Poweshiek County had one, Ottawa City; Keokuk County had two, Western City and West London; Wapello County had several.

Eliminating the 46 towns or villages that have been absorbed by other towns or cities, the 362 that lost their original names by taking on new names, the 119 that had more than one name (as one for the post office and one for the town, or a double name by reason of a nickname), and the 75 that were laid out but never built up, there remain 2,205 actually abandoned placestowns, villages, hamlets, or country post offices.

It will be noticed that in 25 instances the word "Old" appears in parenthesis after the name. This is to distinguish the old and abandoned town from the newer and present one of the same name which is near by, such as Rolfe, Pocahontas County, Rippey, Boone County, and Macedonia, Pottawattamie County. It will also be noticed that 18 names are followed by the letters "P. O." in parenthesis. This is to indicate that the place was a country post office, and to distinguish it from the present town nearby, which was named for the country post office, as Eagle Grove, Wright County, and Defiance, Shelby County. We have paid no attention to the discontinuance of a post office where the village or hamlet is still in active existence.

Here follows the 2,807 names with the counties in which tliey were located: 
Aaronville, Jefierson

Alliyville, Delaware

Alsceum, Jefferson

Acersville, Delaware

Adair, Adair

Adamsonville, Jasper

Adamsville, Davis

Adiville, Plymouth

Addison, Humboldt

Advance, Guthrie

Actni, Wayne

Agency, Winneshiek

Agricola, Mahaska

A jax, Davis

Alaska, Union

Alba, Winnebago

Albany, Davis

Albany, Fayette

Albaton, Monona

Alberton, Jowa

Albriglt's, Des Moines

Aldrich, Wright

Alexandra, Vatn Buren

Algonquin, Butler

Alice, Grundy

Alice, limn

A limeda, Louisa

Alishurg, Webster

Alix, Monlgomery

Allamakee, Allamakec

Allen, Gutlirie

Allen, Harrison

Allen's Grove, Scott

Allenville, Guthrie

Allison, Dubuque

Alma, Dubuque

Alma, Jackson

Almira, Delaware

Almoral, Delaware

Alpine, Wapello

Altamont, Shelhy

Alton, Allamakes

Alton, Dallas

Altona, I.cuisit

Amador, Wapello

Amarill, Delaware

Amazon, Madison

Amboy, Washington
Ambrose, Towa

Aubrosia, I.ce

American City, Marion

Americus, Pottawattamic:

Anish, Johnson

Amity, Pagc

Anity, Scott

Amityville, Des Moines

Amoy, Jackson

Amsterdam, Hancock

Amsterdam, Marion

Amsterdam, Wapello

Amund, Winnebago

Anderson, Clayton

Andersonville, Grumly

Andrews, Polk

Annctta, Woodbury

Annieville, Clay

Antwerp, Cedar

A pollo, Cedar

Apple Grove, Polk

Aquilla Grove, Winmeshick

Arcola, Monoma

Argand, Jones

A rigo, I.ucas

Argo, Scott

A rlington, Montgomery

Armah, Iowa

A rinour, Pottawattanie

Armstrong Grove, Emmet

A rooldsville, Howard

Arrow, Grumdy

Artesian, Bremer

Ashland, Wapello

Ashland Crossing, Wapello

Ashuelot, Kossutl

Ashton, Monoma

Ashurst, Story

Aspinwall, Dubuque

Astoria, Washingtou

Athol, Sioux

Atlanta, Buchanan

Atlec, I.ce

Auburn, Malisski

Auduhon Center, Audubon

Audubon City, Audubon

Audubon I-ieights, Audubon

Aurelia, Floyd 
Aurora, Keokuk Austin, Dickinson Austin, Fremont Avil, Guthric Avon (Old), Polk $\Lambda$ vondale, Adair Ayer's Grove, Polk Ayersville, Wapclio Ayersville, Cedar Babcock, Linn Bach Grove, Wright Baden, Keokuk Badger Hill, liuna Badger Lake, Monona Bailey's Ford, Delaware Bailey's Grove, Hancock Baker, Jefferson Balaka, Clarke Ballard, Audubon Balluff, Scott

Bally Clatugh Grove, Dubugue Baltimore, Henry

Bangall, Henry

Bamner, Warron

Banner Valley, Lim

Barclay, Black Hawk

Barkersville, Marjon

Barrwood, Scott

Barryville, Delaware

Bartiettville, Clarke

Bath, Cerro Gordo

Baum, Cherokee

Baxter (P. O.), Jasper

Baxter, Keokuk

Bay, Delaware

Bear Creek, Poweshick

Bear Creek, Wapello

Bear Grove, Guthrie

Beaver City, Chickasaw

Beaver Grove, Butler

Beebeetown, Harrison

Beelar's Grove, Floyd

Beery, Henry

Beetrace, Appanoóse

Belfast, Henry

Belgrove, Butler

Belinda, Lucas

Bell, Crawford
Bell Air, Johnson

Bell Fountain, Malaska

Bellair, Appanoose

Belle Point, Boone

Bellemont, Warren

Belleville, Jefferson

Belleville, Webster

Bell's lake, Sioux

Bell's Ridge, Madison

Belltown, Crawford

Belmont Center, Warren

Belvidere, Monona

Ben Lomond, Pocahontas

Benan, Carroll

Benbow, Lee

Bennettville, Dubuque

Bennington, Marion

Benson Grove, Winnebago

Benson Grove Station, Winnebago

Benton, Benton

Benton, Mills

Benton Centre, Benton

Benton City, Benton

Bentonville, Wayne

Berea, Adair

Berkeley, Benton

Berlin, Hardin

Berlin, Scott

Bernina, Mahaska

Bethel, Fayette

Bethel; Washington

Bethel City, Marion

Bethlehem, Mills

Bethlehem, Wayne

Beuiah, Benton

Biddiek, Montgomery

Big Grove, Mahaska

Big Grove, Pottawattamic

Big Mound, I.ee

Big Spring, Wayne

liig Springs, Louisit

Big Woods, Jones

Bigler's Grove, Harrison

Binns, Page

Bismarck, Clayton

Bismark, Union

Black Hawk, Davis

Black Hawk, Louisa 
Black Hawk City, Van Buren

Black Walnut, Palo Alto

Blackmore, Ringgold

Bladensburg, Wapello

Blaine, Buena Vista

Blair, Pottawattamie

Blair City, Cherokee

Blairgowrie, Palo Alto

Blakeville, Black Hawk

Blessing, Black Hawk

Bliedorn, Clinton

Bloomefild, Jones

Bloomfield, Monona

Blooming Prairie, Pocahontas

Bloomingdale, Madison

Bloomington, Muscatine

Bloomington, Polk

Bloomington, Ringgold

Bloomington, Story

Blue Cut, Jones

Blue Point, Jefferson

Blue Point, Poweshiek

Bluff Creek, Mahaska

Bluff Creek, Monroe

Bluff Dale, Des Moines

Bluff Point, Monona

Boardman, Story

Bois d'Arc, Grundy

Boltonville, Iowa

Bon Accord, Johnson

Boomer Grove, Pottawattamie

Boone, Dallas

Boone Springs, Clinton

Boonc Station, Boone

Booneville, Boone

Boonsboro, Boone

Border Plains, Webster

Borneo, Ringgold

Botany, Shelby

Botna, Pottawattamie

Bottom, Monona

Boulder, Linn

Bovina, Tuma

Bowen's Prairie, Jones

Boxelder, Mills

Boyd, Chickasaw

Boyer, Pottawattumie

Boyer River, Crawford
Boyer Station, Van Buren

Boyan's Grove, Butler

Boyleston, Henry

Bozaris, Ringgold

Bracewell, Decatur

Bradford, Chickasaw

Brandy Brook, Iinn

Breckinridge, Bremer

Bremen, Monroe

Bremer, Bremer

Bremer Center, Bremer

Brick House, Cedar

Bridgeport, Boone

Bridgeport, Jackson

Bridgeport, Monroe

Bridgeport, Wayne

Bridgewater, Clay

Briscoe, Adams

Bristol, Pottawattamic

Bristol, Worth

Broadway, Delaware

Broadway, Linn

Brock, Mahaska

Broken Kettle, Plymouth

Bromley, Marshall

Brookdale, Plymouth

-Brookfield, Clinton

Brooklin, Polk

Brooklyn, Benton

Brooklyn, Black Hawk

Brooklyn, Calhoun

Brooklyn, Madison

Brookville, Adams

Brookville, Clayton

Brookville, Jefferson

Brough, Dallas

B rown, Linn

Browning, Carroll

Brown's Mills, Davis

Browns Settlement, Woodbury

Brownsville, Poweshiek

Bruce, Wright

Bruner, Tama

Brush Creek, Fayette

Brushy, Taylor

Brushy Valley, Guthrie

Bryson, Wapello

Bubona, Emmet 
Buchanan, Buchanan

Buchanan, Fremont

Buchanan, Taylor

Buchanan, Webster

Buck Creek, Bremer

Buck Horn, Mahaska

Buckeye, Benton

Buckingham (Old), Tama

Buckland, Allamakee

Bucyrus, Lucas

Bucyrus, Mahaska

Buda, Pocahontas

Buena Vista, Clinton

Buena Vista, Harrison

Buenavista, Jefferson

Buffalo, Madison

Buffalo Fork, Kossuth

Buffalo Grove, Buchanan

Bullock's Grove, Pottawattamie

Buncombe, Dubuque

Bunker Hill, Allamakee

Burgess, Clinton

Burke, Benton

Burkhart's Point, Des Moines

Burns, Calhoun

Burr Oak, Mitchell

Burr Oak Grove, Woodbury

Burr Oak Springs, Winneshiek

Burrell, Decatur

Burrington, Delaware

Burris, Louisa

Burris City, Louisa

Burton, Wapello

Bush, Jasper

Business Corners, Van Buren

Busti, Howard

Butler Center, Butler

Butler Hill, Emmet

Butler Rapids, Butler

Butlerville, Tama

Buxton, Monroe

Byron, Humboldt

Cadda, Scott

Calanan, Hamilton

Caldwell City, Appanoose

Caledonia, Dubuque

Caledonia, Ringgold

Calhoun, Appanoose
Calhoun, Calhoun

Calhoun, Harrison

Calhoun Center, Calhoun

Calliope, Sioux

Caloma, Marion

Calvin, Black Hawk

Camackville, Lee

Camargo, Lee

Cambria, Wayne

Cambridge, Clinton

Camden, Appanoose

Camden, Story

Cameron, Cedar

Camp, Polk

Campton, Delaware

Cana, Buchanan

Canaan, Adams

Canfield, Black Hawk

Cannonsburg, Louisa

Canoe, Winneshick

Capoli, Allamakce

Carhon, Webster

Carbon Hill, Davis

Carbonado, Mahaska

Carbondale, Adair

Carbondale, Polk

Cardiff, Mitchell

Carrlisle, Cedar

Carlson, Scott

Carney, Floyd

Carr, Jasper

Carroll, 'Tama

Carroll City, Carroll

Carrollport, Jackson

Carrollton, Carroll

Carr's Point, Montgomery

Carson, Adams

Carson's Point, Boone

Carter, Iowa

Cartersville, Pottawattanie

Carthage, Johnson

Carvers, Allamakee

Cary, Palo Alto

Casady, Webster

Cass, Clayton

Cass Centre, Cass

Cass Centre, Jones

Cassaday's Corners, Boone 
Castana (Old), Monona

Castle Grove, Jones

Castleville, Buchanan

Catteese, Louisa

Cecilia, Howard

Cedar, Cedar

Cedar, Linn

Cedar City, Black Hawk

Cerlar Creek, Greene

Cedar Crossroads, Marshall

Cedar Forks, Sac

Cedar Grove, Iucas

Cedar Mines, Monroc

Cedar River, Cedar

Cedar Town, Muscatine

Cedar Valley, Black Hawk

Cedar Valley, Cedar

Cedarville, Pocahontas

Cedarville, Washington

Centerville, Boone

Centerville, Des Moines

Centerville, Jackson

Centre, Page

Centre Grove, Muscatine

Centre Hill, Washington

Centre 'lown, Dubuque

Centre Valley, Allamakee

Ceres, Clayton

Cerro Gordo, Mills

Cessford, Cedar

Chaldea, Appanoose

Chandler, Keokuk

Chandler, Malaaska

Channingville, Dubuque

Clantry, Allamakee

Chapin (Old), Franklin

Charleston, Jackson

Charlottsville, Madison

Chase, Iohnson

Chatham, Buchatian

Chattanooga, Dallas

Chequest (No. 1), Davis

Cinequest (No. 2), Davis

Cherokce (Old), Cherokee

Cherokee Center, Cherokee

Cherry, Mahaska

Clerry Wood, Clinton

Chester Center, Poweshiek
Chesterfield, Polk

Chicago, Dallas

Chickasaw, Chickasaw

Chickasaw Center, Chickasaw

Chickasaw Station, Chickasaw

Chisholm, Monroe

Christiansburg, Wapello

Churchville, Scott

Cincinnati, Harrison

Circleville, Polk

Civil Bend, Fremont

Civil Point, Audubon

Clanton, Madison

Clarendon, Ida

Clark, Clay

Clark's Ferry, Scott

Clark's Point, Monroe

Clarkstown, Jackson

Clarksville, Monroe

Clay Mill, Jones

Clayford, Jones

Clay's Grove, Lee

Clayton, Pottawattamie

CTear Creek, Allamakee

Clear Lake City, Cerro Gurdo

Clearfield, Poweshiek

Cleveland, Allamakee

Cleveland, Lucas

Clifton, Louisa

Clifton, Winneshiek

Clinton Center, Wayne

Clipper, Ringgold

Cloud, Marion

Clutterville, Butler

Clyde, Jasper

Coal Creek, Keokuk

Coal Valley, Bone

Coalport, Jefferson

Coalton, Monroe

Coaltown, Boone

Cubb, Jackson

Coe's Grove, Montgomery

Coffin's Grove, Delaware

Colbyville, Story

Cold Spring, Pottawattamie

Cold Springs, Cass

Cold Water, Delaware

Coldville, Tama 
Coldwater, Butler

Coldwater, Cerro Gordo

Coldwater, Franklin

College Farm, Story

Collegeton, Story

Collins Centre, Story

Collins Grove, Tama

Coloma, Jackson

Colony, Delaware

Columbia, Tama

Columbia, Wapello

Columbus, Allamakce

Columbus, Linn

Columbus, Van Buren

Comet, Mahaska

Commerce-Mills, Polk

Communia, Clayton

Como, Crawford

Competine, Wapello

Comstock, Wapello

Concert, Mahaska

Concord, Hancock

Concord, Louisa

Condit, Jones

Confidence, Wayne

Congress, Franklin

Conkling, Audubon

Connell, 'Iama

Connor, Allamakec

Cono, Iowa

Conrad Grove, Grundy

Cool, Warren

Coon Grove, Crawford

Coon Valley, Sac

Coonville, Mills

Cooper Springs, Marion

Coopersville, Wapello

Cope, Polk

Copi, Johnson

Coplin's Grove, Carroll

Copper Creek, Jackson

Cora, Decatur

Cora, Des Moines

Cori, Fremont

Cora, Mitchell

Corn Hill, Fayette

Corn Valley, Sioux

Cornelia, Ida
Cory, Keokuk

Corydon, Polk

Cory's Grove, Polk

Cottage, Hardin

Cottage Hill, Dubuque

Cotton Grove, Henry

Cottonville, Jackson

Coulson, Cherokee

Council Bluff, Pottawattamie

Council Hill, Clayton

Council Point, Pottawattamie

Courtwright, Lee

Cousins, Linn

Cox Creek, Clayton

Coyton, Buchanan

Crab Apple, L.inn

Crabb's Mills, Jackson

Craig's Hollow, Webster

Crain Creek, Black Hawk

Crandall, Calhoun

Crane Creek, Howard

Crathorne, Plymouth

Craw ford, Crawford

Cream Hill, Franklin

Creamery, Cerro Gordo

Creesville, Jefferson

Crescent City, Pottawattamie

Cresco, Kossuth

Cressweli, Keokuk

Crete, Appanoosc

Crisp, Harrison

Cromwell Centre, Clay

Crooked Creek, Henry

Crooks, Page

Crooks, Webster

Cross, Ringgold

Crow Creck, Linn

Crown Point, Wright

Crozier, Buena V'ista

Crystal, 'Tama

Cuba, Hancock

Cuba, Monroc

Cuba City, Louisa

Curtis, Johnson .

Cynthiann, Wapello

Cyreno, O'Brien

Dacotal, Dubuue

Daggett, Benton 
Dahlonega, Wapello

Daily City, Boone

Dairy, Washington

Dairy Grove, Jasper

Dairyville, Grundy

Dalby, Allamakee

Dale, Chickasaw

Dalmanutha, Guthrie

Dan, 'laylor

Danforth, Johnson

Danville, Floyd

Danville Center, Des Moines

Darbyville, Appanoose

Darien, Kossuth

Darlington, Sioux

Dartmouth, Jones

Darwin, Page

Dasie, Page

Davis Creek, Washington

Davison's, Page

Dawson, Jasper

Dawsonburg, Fremont.

Dayton, Appanoose

Dayton, Audubon

Dayton, Bremer

Dayton, Iowa

Dayton, Page

Dayton, Story

Dayton, Washington

Dayton Centre, Chickasaw

Daytonville, Washington

De Etta, Poweshiek

Deedsville, Jefferson

Deep liver, Poweshiek

Deer Creek, Fremont

Deer Creek, Worth

Deerfield, Chickasaw

Deering, Winnebago

Defiance (P. O.), Shelby

Defiance, Story

Defiance, Wapello

Delano, Winnebago

Delanta, Hardin

Delaware, Polk

Delaware Centre, Delaware

De I.cen, Cheroket

Del Norte, Davis

Delphi, Marion
Delphi, Ringgold

Dennis, Appanoose

Denson's Ferry, Cedar

Derrinane, Dubuque

Deseret, Pottawattamie

Des Moines City, Mahaska

Des Moines City, Van Buren

Dickey, Bremer

Diff, Appanoose

Discord, Woodbury

Divide, Carroll

Divide, Keokuk

Divide, Marion

Dixie, Jasper

Dixie, Mitchell

Dixonville, Marion

Doantown, Lee

Dodd, Johnson

Dodds, Woodbury

Dodge, Guthrie

Dodgeville, Des Moines

Dogtown, Polk

Dogtown, Van Buren

Donelan, Dubuque

Doran, Mitchell

Dorrville, Warren

Douglas Centre, Clay

Douglass, Fayette

Dover, Davis

Dover, Iowa

Dover, Lee

Dover Mills, Fayette

Downards, Iowa

Downersville, Jones

Downsville, Pottawattamie

Dramen, Mitchell

Draper, Jasper

Dresden, Chickasaw

Dresden, Poweshiek

Dresser, Ida

Drew, Wright

Dry Creek, Linn

Dry Lake, Wright

Dryden, Tama

Duane, Hardin

Duane, Jones

Dublin Washington

Dudley, Polk 
Duggan, Jackson

Duke, Dubuque

Duke, Jackson

Dunnville, Davis

Dureau, Mitchell

Durham's, Marion

Dutch Creek, Washington

Eads, Calhoun

Eads Grove, Delaware

Eagle, Bremer

Eagle City, Hardin

Eagle Grove (P. O.), Wright

Eagle Grove Juct., Wright

Eagle Lake, Kossuth

Eagleville, Wright

Earle, Lucas

East Davenport, Scott

East Grove, Henry

East Hampton, Cherokee

East Melrose, Monroe

East Nodaway, Adams

East Orange, Sioux

East Plattsmouth, Mills

Eastport, Fremont

Eatonville, Howard

Eber, Humboldt

Echo, Harrison

Eden, Benton

Eden, Fayette

Edenville, Marshall

Edinburg, Jones

Edinburg, Union

Edna, Cass

Edom, Keokuk

Edwards, Black Hawk

Edwards, Lee

Egan, Allamakee

Ego, Madison

Egypt, Mills

Eide, Winneshiek

Elba, Carroll

Elbert, Monroe

Elcho, O'Brien

Eldergrove, Allamakee

Eldorado, Decatur

EIdorado, Harrison

Eldorado, Monroe

Eleanor, Butler
Elide, Franklin

Eliza, Black Hawk

Elizabeth, Grundy

Elizabeth City, Scott

Elizabethtown, A ppanoose

Elizabethtown, Cedar

Elizabethtown, Keokuk

Elk, Decatur

Elk Creek, Jasper

Elk Creek, Worth

Elk Grove, Hancock

Elk Rapids, Boone

Elk River, Clinton

Elk Run, Black Hawk

Elk Run, Sac

Elkford, Jones

Elkhart (Old), Polk

Elkhorn (P. O.), Shelby

Elkton, Buena Vista

Ellendale, Plymouth

Ellendale, Warren

Ellenton, Palo Alto

Ellington, Hancock

Elliott, Jasper

Ellis, Hardin

Ellisdale, Pottawattamie

Ellsworth, Madison

Elm Grove, Calhoun

Elm Grove, Marion

Elm Springs, Butler

Elm Springs, Sioux

Elmo, Hancock

Elmont, I.inn

Elrick, Louisa

Ely, Marion

Emert, Black Hawk

Eminence, Pottawattamie

Emmet, Emmet

Emmet Grove, Emmet

Empire, Wright

Energy, Black Hawk

Enfield, Clayton

English Settlement, Marion

Enod, Taylor

Enterprise, Black Hawk

Enterprise, Mahaska

Enterprise, Polk

Ephesus, Dallas 
Erastus, Guthrie

Erie, O'Brien

Erin, Buchanan

Ernest, Lyon

Estella, Ringgold

Ettie, Tama

Eugene, Ringgold

Eureka, Adams

Eureka, Greene

Eureka, 'Tama

Eureka, Washington

Eveland, Mahaska

Eveland Grove, Mahaska

Rvergreen, Dubuque

Evergreen, Tama

Everist, Marion

Excelsior, Mahaska

Factoryville, Dubuque

Fairhaven, Tama

Fairland, Dallas

Fairmount, Marion

Fairplay, Wapello

Fairview, Guthrie

Fairview, Jones

Fairview, Madison

Fairview, Monroe

Fairview, Story

Fairville, Palo Alto

Falls, Dubuque

Fan, Des Moines

Fandon, Allamakee

Farm Creek, Jones

Farmer City, Fremont

Farmers, Sioux

Farmer's Creek, Jackson

Farmersburg, Clayton

Farmersville, Jasper

Farmersville, Mahaska

Farming Grove, Scott

Fayette, Mills

Felix, Warren

Fenton(P. O.), Kossuth

Jern, Grundy

Fern, Worth

Fern Valley, Palo Alto

Ferry, Mahaska

Fierce, Decatur

Fifteen Mile, Tama
Fifteen Mile Grove, 'Tama

Filkin's Grove, Black Hawk

Fine, Kossuth

Fiscus, Audubon

Fisher's Point, Boone

Fisherville, Tama

Flagler, Marion

Flemingville, L.inn

Fletcher, Sac

Flint, Mahaska

Flint Hills, Des Moines

Flood Creek, Floyd

Flora, Montgomery

F'lorence, Benton

Florence, Decatur

Florence, Louisa

Florence City, Black Hawk

Florenceville, Howard

Floyd Center, Floyd

Floyd's Bluff, Woodbury

Flushing, Ringgold

Folger, Clarke

Fontainbleau, Harrison

Fontaine, Hardin

Fontanelle, Worth

Foote, Iowa

Forbes Station, Greene

Forbush, Appanoose

Ford's Grove, Linn

Forest City, Chickasaw

Forest Hill, Louisa

Forest Home, Poweshiek

Forest Mills, Allamakee

Foreston, Howard

Forestville, Delaware

Forks, The, Montgomery

Forks, Tama

Forsyth, Palo Alto

Fort Des Moine (Old), Polk

Fort Plain (No. 1), Warren

Fort Plain (No. 2), Warren

Foster, Madison

Fountain Spring, Wapellu

Four Corners, Jefferson

Fox (No. 1), Davis

Fox (No. 2), Davis

Fox, Iowa

Fox Landing, Des Moines 
Fraker, Monroe

Frankford, Clayton

Frankfort, Montgomery

Frankfort, Webster

Franklin, Clayton

Franklin, Decatur

Franklin, Lee

Franklin, Linn

Franklin Center, Lee

Franklin Grove, Page

Franklin Mills, Des Moines

Franklin Prairie, Winneshiek

Fredonia, Marshall

Fredsville, Grundy

Freedom, Keokuk

Freedom, Lucas

Freehold, Clarke

Freel, Polk

Freeland, Lucas

Freeman, Clay

Freeman, Floyd

Fremont, Benton

Fremont, Iowa

Fremont, Jackson

Fremont City, Fremont

French, Pottawattamie

French Creek, Allamakee

Frenchtown, Black Hawk

Frenchtown, Clayton

Frenchtown, Keokuk

Frendale, Johnson

Friendlings Tavern, Woodbury

Friends' Grove, Warren

Frink's Grove, Buchanan

Fryeburg, Wright

Fuller, Mitchell

Fuller's Mills, Jones

Fulton, Muscatine

Funk's Mills, Decatur

Furray, Plymouth

Gainford, Mahaska

Gainsborough, Van Buren

Gale, Woodbury

Galion, Cass

Galtville, Wright

Galvin, Marshall

Galway, Dallas

Gamble Grove, Fayette
Gambril, Scott

Gamet, Farrison

Gandytown, Davis

Garden, Boone

Garden Grove, Boone

Garfield, Jones

Garibaldi, Keokuk

Garlock, Pocahontas

Garry Owen, Jackson

Gaston, Fremont

Gatesville, Buchanan

Gear, Madison

Gem, Clayton

Geneseo, Cerro Gordo

Geneseo, Tama

Geneva, Benton

Geneva, Muscatine

Genoa, Wayne

Genoa Bluff, Iowa

Georgetown, Butler

Georgetown, Dubuque

Georgetown, Hardin

Georgetown, Monroe

German City, Woodhury

Giard (Old), Clayton

Gibralter, Lyon

Gilbert, Polk

Gilbert, Scott

Gilberttown, Scott

Gilead, Adair

Gillespie, Benton

Gilmantown, Floyd

Gilpin, Madison

Glade, Worth

Glassnevin, Dubuque

Glen Dale, Muscatine

Glen Roy, Howard

Glendale, Scott

Glenmary, Worth

Glenn's, Clarke

Goddard, Jasper

Godfrey, Wapello

Golden Prairie, Delaware

Gomersal, Benton

Gopher, Osceola

Gopher Station, Guthrie

Goshen, Ringgold

Goshen, Story 
Gosport, Marion

Gowando, Marshall

Gower's Ferry, Cedar

Cirace Hill, Washington

Graceville, Guthrie

Giraham, Johnson

Cirainville, Wayne

Grand Gulf, Benton

Grand Meadow, Clayton

Grand River, Wayne

Grand View, Madison

Grange, Woodbury

Grant, Wright

Grantville, Allamakee

Granviile, Mahaska

Gravity (P. O.), Taylor

Gray's Creek, Monroe

Great Oak, Palo Alto

Greeley's Grove, Buchanan

Green, Wapello

Green Bay, Clarke

Green Bay, Lee

Green Bush, Warren

Green Castle, Greene

Green Castle, Johnson

Green Castle, Marshall

Green Hill, Delaware

Green Mountain (Old), Marshall

Green 'Tree, Scott

Green 'I'ree 'Tavern, Scott

Green Valley, Decatur

Greencastle, Jasper

Greene Center, Iowa

Greenops, Greene

- Greensides, Webster

Greenvale, Dallas .

Greenville, Black Iawk

Greenville, Lucas

Greenville, Poweshiek

Greenwood, Chickasaw

Gireenwood, Polk

Greenwood Centre, Kossutlı

Greenwood Hill, Grundy

Greenwood Park, Polk

Gregg, Johnson

Gresham, Black Hawk

Gireys, Greene

Ciriftin, Boone
Griffinsville, Appanoose

Grove, Audubon

Grove, Humboldt

Grove, 'Taylor

Grove, Woodbury

Grove City, Cass

Grove Creek, Delaware

Grove Creek, Jones

Grove Hill, Bremer

Groveland, Adair

Grunwald, Lucis

Grytle, Winnebago

Gurley, Cass

Guss, 'Taylor

Guthrie, Guthrie

Gwinville, Benton

Hackberry, Floyd

Hackney's Grove, Warren

Hagerty, Dickinson

Hale, Kossuth

Hale City, Jones

Hale Village, Jones

Halfway Prairie, Monroe

Hall, Davis

Hall Creek, Monona

Hamnker's, Marion

Hamerville, Buchanan

Hamlin (Old), Audubon

Hamlin, Woodbury

Hamlin Grove, Audubon

Hammondsburgh, Warren

Hancock Center, Hancock

Handsome View, Warren

Hanover, Allamakee

Hanover, Polk

Happy Corners, Plymouth

Happy Hollow, Wapello

Happy Hollow, Wayne:

Harbor, Davis

Habor Station, Gutlrie

Harder's Corner, Page

Hardin, Allamakee

Hardin, Clayton

Hardin, Greene

Iardin City, I-Iardin

Harmon's Switch, Boone

Harnony, Jefferson

Harmony, 'Iaylor' 
Harpersville, Davis

Harris Grove, Harrison

Harrisburg, Mahaska

Harrisburg (No. 1), Van Buren

Harrisburg (No. 2), Van Buren

Harrisburg, Washington

Harrison, Guthrie

Harrison, Harrison

Harrison, Louisa

Harrison, Madison

Harrison, Pottawattamie

Harrisonville, Marion

I-Iartford, Des Moines

Hartland, Marshall

Hartland, Worth

Hartwick, Delaware

Harvard, Wayne

Harvey Mills, Dallas

Harvey's Mills, Jefferson

Harvey's Point, Polk

Harwell, Cedar

Haskalia, Webster

Hatch, Kossuth

Hatton, Polk

Hauntown, Clinton

Havanna, Greene

Haven, Tama

Hawkeye, Des Moines

Hawkeye City, Union

Hawthorn, Montgomery

Hayes, Adams

Hazard, Cherokee

Hazel, Dubuque

Hazel Green, Deliwware

Hiazel Green, Hairdin

Hazen, Lee

Hazleton (Old), Buchanan

Heath, Tama

Heaton, Madison

Hedge, Iowa

Hedge's Grove, Cass

Hedrolante, Van Buren

Helena, Tama

Hempstead, Dubuque

Henderson, Lucas

Henderson Prairie, Clayton

Henn, Monrue

Henness, Mahaska
Henshaw, Taylor

Henton, Mills

Hentonville, Mills

Herdland, Clay

Hesperian, Webster

Hiawatha, Monona

Hibbsville, Appanoose

Hickory, Van Buren

Hickory Grove, Clarke

Hickory Grove, Jackson

Hickory Grove, Monroe

Hickory Grove, Scott

Hickory Point, Clarke

Higginsport, Dubuque

Higginsport, Jackson

High Creek, Fremont

High Grove, Clayton

High Lake, Emmet

Highbrier, Decatur

Highland, Clayton

Highland, Union

Highland City, Pocahontas

Highland Grove, Jones

Highland Park, Sioux

Hillsboro, Louisa

Hillsdale, Mills

Hilltown, Appanoose

Hilton, Monroe

Hinkletown, Iowa

Hires Grove, Buena Vista

Hirondelle, Worth

Hitesville, Butler

Hobart, Kossuth

Hodge, Wayne

Hoenzollern, Crawford

Hogansville, Dubuque

Holaday's, Adair

Hollandale, Winnebago

Hollenback, Linn

Holley, Plymouth

Hollidaysburg, Monroe

Holman, Osceola

Holmstad, Montgomery

Holt, 'Taylor

Homan, Tama

Home, Van Buren

Homer, Hamilton

Honey Creek, Clayton 
Honey Grove, Cedar

Iook's Point, Hamilton

Hoosier Grove, I.inn

Hoosier Mills, I.inn

Hoosier Point, Benton

Hope Farm, Louisa

Hopewell, Mahaskal

Hopkins Grove, Polk

Hoprig, Emmet

Horace, Audubon

Horn, Jasper

Hornbuckles Point, Boone

Horton, Bremer

Hoskins, Woodbury

Jotona, Tama

Howard, Howard

Howard, 'lama

Howard Center, Allamakee

Howard Centre, Howard

Howardville, Floyd

Howsier, Harrison

loyt, Adams

Hudsen, Muscatine

Hueston, Johnson

Hugo, Jackson

Hull, Boone

Humboldt (P. O.), Humboldt

Tumbug Town, Poweshiek

Hummaconna, Mon roe

Hungarian Settlement, Decatur

Huntsville, Polk

Huron, Des Moines

Husan, Scott

Hustons, Keokuk

Hyde, Winneshiek

Hynes, Monroe

Icaria, Adams

Iconium, A ppanoose

Ida, Ida

Idaho, Hardin

Idlewild, Buchanan

Illinois Grove, Marshall

Illyria, Fayette

Incline, Boone

Independence, Decatur

Independence, Madisun

Independence Centre, Jasper

Independent, $V$ an Buren
Indian Creek, Mills

Indian Prairie, Van Buren

Indian 'lown, Tama

Indian Village, Johnson

Indiana, Marion

Indianapolis, Mahaska

Indiantown, Cass

Tndiantown, Chickasaw

Ingarts, Ringgold

Ingart's Grove, Ringurold

Ingham, Franklin

Ingleville, Clay

Ingraham, Mills

Inland, Cedar

Ioka, Keokuk

Iola, Marion

Iola, Pottawattamie

Ione, Iowa

Iowa, Muscatine

Iowa Center, Story

Towa City, Vin Buren

Towa Lake, Emmet

Towa 'lown, I.umisa

Iowaville, Van Buren

Iranistan, Cass

Irene, Sioux

Irish Grove, I.ucas

Irish Grove, Warren

Iron Hill, Jackson

Irving (Old), 'Tama

Irvingtón (Old), Kossuth

Irwin, Audubon

Isabell, Jones

Isabella, Jackson

Island Grove, Butler

Island Grove, Enmet

Isted, Winneshiek

Iten, Shelby

Iuka, Tyon

Iuka, 'Tama.

Ivanhoe, I.inn

Ives, Johnson

Ives, Palo Alto

Ivester, Grundy

Iveyville, Adams

Jack Creck, Clarke

Jackson, Adair

Jackson Center, Webster 
Jackson Point, Bremer Jacksonville, Chickasaw Jacksonville, Clarke Jacksonville, Clayton Jacksonville, Des Moines Jacobs, Poweshiek Janestown, Howard Janestown, Scott Jannestown, Winneshiek .Janesville, Tama Janetown, Keokuk Jasper, Carroll Jasper City, Jasper Jeddo City, Harrison Jefferson, Clayton Jefferson, Dubuque Jefferson, Fayette Jefferson, Henry Jefferson City, Bremer Jeffersonville, Lee Jehu, Boone Jenkins Center, Mitchell Jenks, Taylor Jerico, Chickasaw Jerico, Polk Jeromeville, Marshall Jerusalem, Grundy Jewell, Mills Jobe's, Audubon Johns, Appanoose Johnson, Jones Johnson Settlement, Kossuth Johnsonport, Allamakee Johnson's Grove, Story Johnsontown, Jones Johnsonville, Crawford Johnston, Appanoose Jollyville, L.ee Jones, Iowa Jonesville, Jones Toy, Plymouth Juan, Washington Jubilee, Black Hawk Junction City, Mills Kalo Junction, Webster Kane, Pottawattamie Kanesville, Pottawattamie Kars, Tama
Kasson, Madison

Keb, Wapello

Keelroy, Clayton

Keen, Polk

Keensburg, Keokuk

Keg Creek, Pottawattamie Kelsey, Polk

Kemigala, Appanoose

Kemling, Pottawattamie

Kendrick, Greene

Kentner, Carroll

Kentuck Grove, Hamilton

Keown, Pottawattamie

Kerr, Scott

Kesho, Webster

Kew, Ringgold

Key, Bremer

Kier, Buchanan

Kimball, Jasper

King, Dubuque

Kings, Union

Kingsbury, Grundy

Kingston, Linn

Kingston City, Linn

Kinisaw, Tama

Kirkwood, Appanoose

Kirkwood, Polk

Kiron (Old), Craw ford

Kissemmee, Calhoun

Kline, Des Moines

Klinger, Bremer

Kniffin, Wayne

Knittel, Bremer

Knowlton, Ringgold

Knox, Black Hawk

Knox, Fremont

Kossuth, Des Moines

Kossuth Centre, Kossuth

Krogness, Lyon

Krum, Jefferson

Lacey, Muscatine

Lackawana, Webster

I.acona, Hancock

Lactin, Cedar

I.afayette, Allamakee

Lafayette, Keokuk

Laf ayette, Linn

Lafayette, Louisa 
Lif fayctte, Marshall Lafayette, Polk

I.a Grange, Lucas

l.a Grange, Monroe

La Harp, Clarke

I.ahart, Monroe

la Hoyt, Henry

I,ake, Washington

I ake Center, Hamilton

Lake Prairic, Marion

Lake View, Palo Alto

lakeport, Woodbury

Lakeside, Emmet

Lakeville, Dickinson

I.akin's Grove, Hamilton

L.ancaster, Henry

I.ancaster, Keokuk

I.andis, Taylor

Laneville, Appanoose

l.angfitt, Dallas

Lansrud, Worth

Laporte, Clarke

Laredo, Mahaska

Lark, Worth

Larland, Audubon

Last Chance, Lucas

l.atham, Webster

Latimer, Warren

lattimer's Grove, Poweshick

L.al Vega, Des Moincs

Lavega, Madison

Lawrence, Fremont

l.aw rence, Mills

Lawrenceburg, Warren

La Yuba, Hardin

Leadville, Guthrie

L.car, Grundy

l.carned, Pocahontas

l.eath City, Adair

L.ebanon, Appanoose

L.eClair Centre, Scott

Lee, Ringgold

Lec, Union

Leerdam, Marion

Lectown, Appanoose

Lefevre, Madison

Lelandsburgh, Winnebago

Lemont, Adair
Leo, Fayette

L.eo, Mitchell

Jeona, Appanoose

Leona, Johnson

Leonard, Taylor

Leoni, Butler

Leota, Sac

L.eroy, Bremer

Leroyville, Auduboin

Lesan, Ringgold

Lester, Black Hawk

Lewis City, Mills

Lewishurg, Wayne

Lewiston, Davis

Lewiston, Winnebayo

Lexington, Iones

Lexington, Osceola

Lexington, Taylor

Lexington, Van Buren

Lexington, Washington

Liberty, Marshall

Liberty, Wright

Lick Creek, Van Buren

Lightville, Fayette

Likens, Benton

Lilly, Pocahontas

Lima, Pottawattamic

L.ime City, Cedar

Lime Creek, Cerro Gordo

Lime Creek City, Winnebago

Lime Springs (Old), Howard

Limestone, Des Moines

Lincoln, Grundy

Lincoln, Polk

Lincoln Center, Grundy

Lincoln Centre, Mitchell

Lindon, Linn

Lindville, Monroe

Link, Hardin

Linn, Cerro Gurdo

Linn, Dallas

Linn, Lucas

Linn Grove, Scott

Linnburg, Webster

Linndale, Winneshick

Linton, Des Moines

Linwood, Adair

Linwood, Benton 
Linwood, Scott

Listonville, Woodlbury

L.itchifield, 'Taylor

Lithgow, Cedar

L.ithopolis, Hardin

Little River, Decatur

Little Turkey, Chickasaw

Littleton, Buchinan

Living Spring, Pottawattamie

L.ivingstone, Appanoose

Livonia, Cerro Gordo

Lizatrd, Cedar

Lizard, Pocahontas

Lockridge, Jefferson

Locust Hill, Clayton

Locust Lanc, Winneshiek

Lodo Mello, Delaware

Lodomillo, Clayton

Logansport, Boone

L.omlard, Pocalontas

London, Benton

Lone Office, 'Iaylor

Long Creck, Decatur

Long Creek Station, Lous:sa

Longview, Van Buren

Lore, Dubuque

Loretto, Warren

Loring, Polk

Losh's Mills, Pottawattamie

Lossing, Monona

Lost Creek, Mahaska

Lost Island, Palo Alto

Lotts, Humboldt

Louden, Mills

Louisa Center, Louisa

Louise, Black Hawk

Louisville, Audubon

Louisville, Fayctte

Louisville, Wapello

L.ovington, Polk

Lowell, Butler

Lowell, Jackson

Lowther Station, Howard

loy, Page

I.ozier, Woodbury

I.ucas, Muscatine

Lucas Grove, Marion

Lucerne, Wayne
Lucky Valley, Wuodbury

I.udlow, Allamakee

L.uella, Pocahontas

Lunds, Webster

J.uni, Wright

L.unsford, Davis

I.ura, Cass

Lutra, Fayette

Lybrand, Allamakee

I.ydia, Crawford

I.yndale, Allamakee

I.ynn, Warren

L.yons, Clinton

L.y tle City, Iowa

McBride, Madison

MeCabes, Henry

McCants, Dubuque

McCarverstown, Henry

MeDevitt's Grove, Polk

McDill, Catss

McDill, Union

Macedonia (Old), Pottawattamie

Maceville, Lucas

McGonigle, Linn

McGregor's Landing, Clayton

MeGuire, Webster

McKily, Dallas

Mackey, Boone

Mackey's Grove, Boone

Mckinley, Adair

Mckissick's Grove, Fremont

McKnight's Puint, Humboldt

Macks, Carroll

Macksville, Guthrie

McLaughlin's Creek, Webster

McPherson, Madison .

McVeigh, Van Buren

Madison, Jones

Madison, Lee

Malhaska, Mallaska

Maidia, Guthrie

Mahern, Johnson

Makee, Allamakee

Mallory, Shelby

Malta, Marshall

Malton, Plymouth

Mammen, Plymouth

Manat, Poweshiek 
Manathekil, Benton

Manchester, Adair

Manchester, Allamakec

Mangoldville, Delaware

Manlattan, Keokuk

Mansfield, Lucas

Manteno, Shelby

Manti, Fremont

Mantua, Monroc

Maple, Cherokce

Maple Grove, Madison

Mapleton (Old), Monona

Mapleville, Chickasaw

Marithon, Shelby

Marcellus, Washington

Marcy, Boone

Mardensville, Appanoose

Marena, Ringgold

Marietta, Marshall

Mariner's Hope, Allamakec

Marion, Hamilton

Marion, Wapello

Marshall, Henry

Marshall, Marshall

Marshall, Ringgold

Marshalitown, Ringgold

Marshfield, Dubuque

Marshfield, Jones

Narten's Ranch, Harrison

Martinsburg, Bremer

Martinsburg, Davis

Martinsville, Davis

Marvin; Pocahontas

Marysville, Benton

Marysuille, Wapello

Marysville, Winneshiek

Masonic Grove, Cerro Gordo

Massilon, Fayette

Maunch Chunk, Mahaska

Maudville, Butler

Maulshy, Marshall

Maurice, Sioux

Naxfield, Bremer

May Bell, Sioux

May City, Osceola

Malyficld, Linn

Maysville, Franklin

Mayview, Buena Vista
Mear, Clinton

Meade, Page

Mechanicsburg, Van Burcn

Mechanicshurg, Wapello

Medford, Warren

Meeks Mills, Vin Buren

Meirotto, Lee

Melhourne, Plymouth

Melpine, Muscatine

Melrose, Grundy

Melrose, Harrison

Melrose, Lee

Melville, Audubon

Memory, Taylor

Memphis, Ajpanoose

Mennon, Matrion

Menoti, Buena Vista

Mentor, Bremer

Menzic, Franklin

Mercer, Adams

Mercer, Sác

Merrill, Marion

Merrimac, Jefferson

Miama, Monroe

Micl Prairie, Louisa

Middle River, Madison

Middlelourg, Washington

Middlefield, Buchanan

Middleport, Clarke

Middletown, Mahaska

Middletown, Scott

Midland, Hardin

Midland, Tama

Midlothian, Wapello

Nidwaty, Boone

Midway, Polk

Nidway, Woodbury

Mikesville, Hancock

Milan, Keokuk

Milan, Lucis

Milford, Clarke

Milford, Decitur

Milford (Old), Dickinson

Milford, Montgomery

Mill, Fayette

Mill Grove, Fayette

Mill Grove, Powcshick

Millard, Appanoose 
Milledgeville, Appanoose

Milleray, Dubuque

Miller's, Dubuque

Miller's Creek, Black Hawk

Miller's Hollow, Pottawattamie

Millheim, Delaware

Mill rock, Jackson

Mills, Washington

Milo, Delaware

Milton, Allamakee

Milton, Jefferson

Milton, Pocahontas

Milton Station, Mills

Mineral Creck, Jones

Mineral Ridge, Boone

Minerstown, Monroc

Minerva (P. O.), Marshall

Minkler, Bremer

Minnabrook, Plymouth

Minnic, Dickinson

Mitchell, Polk

Moffiitt's Grove, Guthrie

Mondieu, Linn

Moneek, Winneshiek

Monmouth, Butler

Monroe, Jefferson

Monroe, Johnson

Monroe City, Jasper

Montacute, Polk

Montana, Boone

Montgomery, Wright

Monti, Buchanan

Monticello, 'Tama

Montpelier, Madison

Montpelier (Old), Muscatine

Montpelier, Warren

Mooreville, 'lama

Morgan, Crawford

Morgan, Decatur

Morgan, Wimneshiek

Morgan Valley, Marion

Morfordsville, Jolnson

Morhain, Wright

Moriah, Iowa

Mormon Hill, Marshall

Normontown, Taylor

Morris, Woodbury

Morrisburg, Guthrie
Morristown, Jasper

Morsman, Page

Mortimer, Ringgold

Morton, Pottawattamie

Musalem, Dubuque

Moscow, Wright

Mosley, Calhoun

Motor, Warren

Mount Algor, Jackson

Mount Auburn, Polk

Mount Calvary, Davis

Mount Gilead, $\Lambda_{\text {ppanoose }}$

Mount Hope, Delaware

Mount Hope, Sac

Mount Norris, Harrison

Mount Olive, Mills

Mount Pisgah, Union

Mount Sterling, Jefferson

Mount Sterling, Louisa

Mount Valley, Winncbago

Mount Vernon, Mahaska

Mount Washington, Adams

Muchachinock, Mahaska

Muddy, Calhoun

Mudville, Buchanan

Mullarky's Grove, Black Hawk

Munn, Cedar

Munterville, Wapello

Muscatine, Keokuk

Musquaka, Iowa

Myers, Union

Myron, Allamakee

Myrtle, Muscatine

Nanisee, Wapello

Nanito, Adair

Nansen, Chickasaw

Nanson, Pottawattamic

Nantville, Black Hawk

Napoleon, Johnson

Napeoleon, Van Buren

Narrows, Madison

Nasheim, Winnebago

Nashville, Lce

Nassau, Keokuk

Navarre, Des Moines

Nebraska, Pottawattamie

Nebraska, Webster

Necot, Linn. 
Needmore, Harrison

Nekima, Sioux

Nelson, Guthrie

Nelson, Mitchell

Nemora, Johnson

Neptune, Plymouth

Neutral, Bremer

Nevada, Iowa

New Alba, Winneshiek

New Albany, Sac

New Albany, Story

New Albion, Butler

New Bergen, Emmet

New Buda, Decatur

New Buffalo, Linn

New Buffalo, Scott

New Buffalo, Woorlbury

New Castlc, Jackson

New Castle, Jolnson

New Castle, Marion

New Cherokee, Cherokee

New Chicago, Marion

New Chicago, Ringgold

New Galena, Allamakec

New Hamburg, Scott

New Haven, Jefferson

New Haven, Washington

New Hope, Appanoose

New Hope, Union

New Ireland, Dallas

New Jefferson, Grecne

New Jerusalem, Butler

New Lexington, Van Burcn

$\mathrm{New}$ Lindon, Linn

New Market, Van Buren

New Munich, Sac

New Oregon, Howard

New Philadelphia, Story

New Plains, Appanoose

New Port, Ringgold

New Saint Johns, Harrison

New Stand, Clayton

New 'Town, Marion

New Town, Pottawattamic

New Vine, Dubuque

New Tork, Clinton

New York, Wayne

Niewark, Linn
Newark, Marion

Newark, Wchster

Newburg, Muscatine

Newhurg, Mitchell

Newlon's Grove, Cass

Newport, Jones

Newport Center, Johnson

Newton, Keokuk

Newton City, Jisper

Newtonville, Buchanan

Nezcka, Allamakee

Niles, Appanoose

Niles, Floyd

Niles, Van Buren

Niles Grove, Carroll

Nine Eagles, Decatur

Nine Mile, Mahaska

Nishma, Pottawattanic

Noblesville, Davis

Nobleton, Polk

Nodaway, Page

Nodaway Fork, Page

Nodaway Mills, Page

Noebli, Kossuth

Nora, Humboldt

Nordland, Worth

Nordyke, Dillas

Normiln, Winneliago

Norris, Clarke

Norris, Marshall

North, Madison

North Bend, Mitchell

North Bentonsport, Van Buren

North Branch, Madison

Nortl Capoli, Allamakec

North Des Moines, Polk

North Grove, Patge

North McGregor, Clayton

North Maquoketa, Jackson

North Mills, Page

North River, Madison

North Skunk, Poweshick

North Skunk River, Jasper

Northam, Bucna Vista

Northfield, Des Moines

Northport, Benton

Northville, Greene

Nortonville, Clarke 
Norway, Fayette

Norwich, Jones

Norwich, Wright

Noseville, Davis

Nottingham, Davis

Nottingham, Delaware

Nugent's Grove, I.inn

Nunda, Fremont

Oak, Marion

Oak Grove, Iinn

Oak Point, Van Buren

Oak Spring, Davis

Oakfield, Audubon

Oakland, Clarke

Oakland, Delaware

Oakland, Dubuque

Oakland, Louisa

Oakland, Van Buren

Oakland Valley, Franklin

Oakvale, Worth

Oakwood, Polk

Oakwood, Tama

O'Brien, O'Brien

Ocheycdan (P. O.), Osceola

Odd, Woodbury

Odessa, Louisa

Ogden, Dubuque

Ohio, Madison

Ohio, Marion

Ojedo, Harrison

Ola, Lucas

Ola, 'Tama

Old Mission, Winneshiek

Old Saint Johns, Harrison

Old 'lown, Palo Alto

Oldfield, Polk

Olean, Keokuk

O'Leary, Plymouth

Olio, Union

Oliver, Sac

Olivet, Union

Olmitz, Lucis

Olmstead, Harrison

Olympus, Harrison

Onawa City, Monona

Oneska, Mahaska

Onion Grove, Cedar

Ononwa, Louisa
Opposition, Wapello

Oradell, Marion

Oran, Fayette

Orange, Clinton

Ord, Madison

Orford, Tama

Orion, Fayette

Orlando, Wayne

Orleans, Appanoose

Orleans, Winneshiek

Ormanville, Wapello

Ornbaum, Malıaska

Oro, Montgomery

Orono, Muscatine

Orrin Glen, Delaware

Orrinden, Delaware

Orrville, Keokuk

Orrville, Mitchell

Orsland, Buena Vista

Orville, Appanoose

Osage, Fremont

Osborn, Howard

Oshkosh, Sioux

Oskaloosa Junction, Malıaska

Osprey, Dallas

Osprey, Monroe

Oswalt, Jasper

Oswegro, Warren

Otho (P. O.), Webster

Otisville, Franklin

Otisville, W right

Otranto (Old), Mitchell

Otsego, Fayctte

Otsego, Wright

Ottawa, Black Hawk

Ottawil, Clarke

Ottawa, Polk

Ottawa City, Poweshiek

Otter Creek, Criawford

Otter Creek, Jackson

Otter Creek, Limm

Otter Creck, 'Tima

Otterville, Buchanan

Overmans Ferry, Muscatine

Overton, Lee

Ovid, Taylor

Ovid, Wayne

Owen, Cerro Gordo 
Owen's Grove, Cerro Gurdo

Owlake, Humboldt

Oxford, Mahaska

Ozark, Jackson

Pacific, Pottawattamic

Pacific City, Mills

Paddington, Linn

Paduca, Greene

Paducah, Boone

Page City, Page

Paint Creek Valley, Allamaket

Paint Rock, Allamakee

Palermo, Grundy

Palestine, Johnson

Palestine, Story

Palestine, Wapello

Palestine, Van Buren

Palmer, Polk

Palinyra, Jasper

Palo Alto, Louisa

Palsville, Wright

Pamaho, Jones

Panneko, Van Buren

Panther, Dallas

Panther Creek, Clayton

Paoli, Palo Alto

Paradise, Crawford

Paran City, Marion

Paris, Decatur

Paris, Mamilton

Paris, Washington

Park, I.yon

l'ark Grove, Humboldt

Parkershurg, Boune

Parkershurg, Jasper

Parliersville, Van Buren

Parkhurst, Scott

Parma, Pottawattamie

Parrish, Des Moines

Parrish City, Harrison

larsonsville, Jefterson

Parvin, Pocaliontas

Patriot, Decatur

Pitriot, Union

Pattersonville, Sioux

Paul, Benton

Peach, Buena. Vista

Pearl City, Appanoose
Pearl Rock, Chickasaw

Peck's, Keokuk

Peck's Ferry, Clayton

Pedec, Cedar

Pekay, Mahaska

Peioro, Woodbury

Pelton, Poik

Penn, Fayette

Pemnington, Lyon

Pennsburg, Guthrie,

Penoach, Dallas

Peoria, Wayno

Peorial City, Polk

Perryilile, Marion

Peru, Dubuque

Peru (Olal), Madison

Petershurg, Kcokuk

Petersburg, Polk

Petersville, Union

Pettis, Salc

Phialanx, Mahiska

Philadelphia, Van Buren

Philhy, O'Brien

Phillips, Wapellu

Phillipston, Bremer

Plilo, Sac

Philo, Union

Pickaway, Benton

P'ickwick, Wapello

Pierce, Jones

Pierce Point, Dallas

Pigeon, Pottawattamie

Pike, Muscatine

Pilgrims Grove, Hardin

Pilot Rock, Cherokee

Pilothurg, Washington

Pin Oik, Dubuque

Pinacle Point, Scott

linchey, Marion

line, Buchanan

Pine Mils, Muscitine

Pinlook, Linn

Pioncer Grove, Cedar

Pisgal, Union

Pittsburg, Louisa

Pitzer, Madison

Plainview, Scott

Planeville, Warren 
Platt, Clay

Platte, 'Taylor

Platteville, Taylor

Plattville, Mills

Pleasant Corners, Monroe

Pleasant Grove, Des Moines

Pleasant Grove, Jefferson

Pleasant Grove, Keokuk

Pleasant Hill, Cedar

Pleasant Hill, Keokuk

Pleasant Hill, Van Buren

Pleasant Lane, Wapello

Pleasant Plain, Decatur

Pleasant View, Appanoose

Pleasant View, Davis

Pleasant View, Humboldt

Pleasant View, Jasper

Pleasant View, Madison

Plcasantville, Appanoose

Plow, Hamilton

Plum, Buenà Vista

Plum Creek, Delaware

Plum Creek, Dubuque

Plum Hollow, Fremont

Plum Spring, Delaware

Plymoutl, Plymouth

Piymouth, Van Buren

Plymouth Centre, Plymouth

Plymouth City, Plymouth

Plymouth Rock, Winneshiek

Pocahontas Center, Pocahontas

Point Isabel, Wapello

Point Palestine, Story

Point Pleasant, Hardin

Point Pleasant, Scott

Poland's Grove, Hamilton

Polen, Ringgold

Polk, Bremer

Polk, Lucas

Polk, Mahaska

Polo Station, Worth

Pomo, Shelby

Pond Valley, Howard

Ponona, Plymouth

Pony, Bremer

Poplar, Audubon

Poplar Grove, Hamilton

Poplar Grove, Marion
Poplar Grove, Palo Alto

Port Allen, Louisa

Port Allen, Musentine

Port Louisa, Louisa

Port Richmond, Wapello

Port Washington, Louisa

Porters, Webster

Portland, Muscatine

Portland, Van Buren

Portlandville, Plymouth

Portoro, Van Buren

Potato Hill, Benton

Potosia, Plymouth

Pottawattamie, Pottawattamie

Potter, Mills

Potter, 'Tami

Pottsville, Washington

Poughkeepsie, Hardin

Poultney, Delaware

Powashcek, Muscatine

Powhattan, Pocahontas

Poyneer, Ringgold

Prairie City, Decatur

Prairic City, Story

Prairic Creek, Benton

Prairic Creek, Dubuque

Prairie Creek, Iowa

Prairie Flower, Pottawattamie

Prairie Grove, Clarke

Prairie Hill, Boone

Prairie House, Lee

Prairie La Porte, Clayton

Prairic Mills, Muscatine

Prairic Rose, Shelby

Prairie Springs, Jackson

Prairie View, Ringgold

Prairiebelle, Jasper

Prairieville, Decatur

Preparation (Old), Monona

Price, Madison

Price, Scott

Princeton, Monroe

Prospect, Clay

Prospect Hill, I.inn

Prosper, Butler

Prussia, Adair

Prussia Center, Adair

Putnam, Fayette 
Pymosa, Cass

Pyra, Warren

Quebec, Hardin

Queen City, Adums

Queen's Point, Madison

Quigley, Clinton

Quincy, Adams

Quincy, Boone

Quincy, Jasper

Quinn's Grove, Ringgold

Quorn, Plymouth

Raccoon Forks, Polk

Raccoon River, Polk

Racine, Buena Vista.

Raglan, Harrison

Rahm, Kossuth

Raleigh, Emmet

Ramesse, Clinton

Ramona, Tama

Ramsey, Kossuth

Randolph, Humboldt

Rankin City, Davis

Ransom, Monona

Rantout, Shelby

Rapids, Boone

Rasbach, Plymouth

Rater, Davis

Rathton, Hardin

Ratna, Winnebago

Ray, Appanoose

Read, Clayton

Red Cedar, Hamilton

Red Cedar Mills, Marion

Red Oak, Cedar

Red Oak Junction, Montgomery

Redding, Ringgold

Redliead, Polk

Redman, Tama

Reed, Madison

Reeder's Mills, Harrison

Reed's Ridge, Allamakee

Reedsville, Marshall

Reedville, Marion

Reels, Pottawattamie

Reeve, Franklin

Renner, Boone

Reno, Cass

Republic, Chickasaw
Reynolds, Kossuth

Rhoads, Taylor

Riclfield, Fayette

Richland, Jones

Richmond, Davis

Richmond, Madison

Richmond, Wapello

Ricker, Sioux

Ricord, Hardin

Ridgedale, l'olk

Ridgeport, Boone

Ridgeway, Guthrie

Riley, Clarke

Ringgold, Ringgold

Ringgold City, Ringgold

Ringsted (P. O.), Emmet.

Ringwood, Clinton

Ripley, Floyd

Rippey (Old), Greene

Rising Sun, Appanoose

Rising Sun, Van Buren

River Side, Boone

River View, Lyon

Riverdale, Iowa

Riverton, Floyd

Roach, Shelby

Robin, Benton

Robinson, Delaware

Rochdale, Shelby

Rochelle, Adams

Rochester, Mahaska

Rochester, Van Buren

Rock, Cerro Gordo

Rock Branch, Woodbury

Rock Creek, Cedar

Rock Creek, Mitchell

Rock Grove City, Floyd

Rock Mills, Sioux

Rock Point, Cedar

Rock Run, Slıelby

Rockingham, Scott

Rocksylvania, Hardin

Rockton, Marshall

Rockville, Delaware

Rockwood, Hardin

Rode, Harrison

Rogerville, Benton

Rolfe (Old), Pocahontas 
Rolfe Junction, Pocahontas

Rolley, Jackson

Romano, O'Brien

Rome, Jones

Root's Siding, Butler

Roscoe, Davis

Rose Grove, Hamilton

Rose Hill (P. O.), Mahaska.

Rosedale, Wright

Roselle, Carroll

Rosemount, Warren

Rosendale, Hamilton

Rosetta, Keokuk

Rosette, Cedar

Roseville, Floyd

Ross Grove, Montgomery

Rossville, Montgomery

Round Grove, Scott

Rousseau, Marion

Roxie, Bremer

Royal Ridre, Sioux

Rubens, Pucalontats

Runyan, Osceola

Rural, Henry

Rural, I.inn

Ruslı Lake, Osceola

Rush Lake, Pilo Alto

Rushviile, Jasper

Rushville, Mills

Rusk, Pocahontas

Russelville, Lee

Sac \& Fox Agency, Wapello

Safeside, Guthrie

Sage, Palo Nlto

Sailorville, Wapello

Saint Charles City, Floyd

Saint Clair, Mononat

Saint George, Monoma

Saint Gilman, Osceola

Saint Jolan, Clayton

Saint Johns, Harrison

Saint Joseph's Prairie, Duhuque

Saint Julien, Linn

Saint Mary's, Iinn

Saint Mary`s, Mills

Saint Paul Junction, Plymouth

Salem, Muscatine

Salt Creek, Davis
Salt Crcek, Tama

Salubria, Van Buren

Samison, Shelby

Sampson, Winneshick

Samville, Wayne

Sanbornton, Clinton

Sand Creek, Delaware

Sand Ridge, Des Moines

Sandersville, Hardin

Sandyville, Warren

Saratoga, Hamilton

Sargent, Floyd

Salude, Chickasaw

Saxon, Wayne

Sityre, Buena Vista

Schoharie, Sac

Schonberg, Calhoun

Schonberg, Warren

Sciola, Montgonery

Scott, Fayette

Scott, Fluyd

Scott, Mahaska

Scott Center, Fayette

Scottswood, Pottawattamie

Seranton City, Greenc:

Scranton Station, Greenc

Scaton, Fayette

Sedalia, Ciuthrie

Sedgrewick, Decatur

Seevers, Jasper

Seigel, Bremer

Selection, Monroe

Selma, Wayne

Sepanamo, Johnson

Sevastopol, Polk

Seventy-eight, Johnson

Seventy-seven, Johnson

Seventy-six Center, Washington

Slabhona, O'Brien

Shady Brook, Timn

Shady Grove, Buchaman

Slaron (Old), Appinoose

Sharon, Warren

Sharps, Taylor

Sharpsburg, Mills

Sharpiston, Appinoose

Shawrille, $\Lambda$ ppanoost:

Sheffield, Dubuque 
Sheffield, Story

Shelby's, Clarke

Shelbyville, Shelby

Shell Rock, Woodbury

Shell Rock Falls, Cerro Gordo

Sheridan, Poweshiek

Sheridan, Sioux

Sheridan, Van Buren

Sheridan Center, Poweshiek

Sherlock, Palo Alto

Sherman, Poweshick

Sherman Center, Montgomery

Shiloh, Cedar

Shinn, Adams

Shirley, Pocahontas

Shobe's Grove, Franklin

Shockley, Henry

Shuck's Station, Wapello

Shoo Fly, Johnson

Showman, Keokuk

Siam, 'Taylor

Sickles, Wapello

Side Hill, Cedar

Sigrel, Cliayton

Silshe, Jackson

Silver Creek, Delaware

Silver Creek, Ida

Silver Creek, Pottawattamie

Silver Lake, Worth

Silver Street, Ringgold

Simpson, Adams

Sisley`s Grove, Linn

Slabtown, Webster

Slagle, Keokuk

Smiyville, Story

Smead, Lyon

Smithfield, Allamakee

Smithfield, Story

Smitl's Ferry, Jackson

Smitl's Point, Boone

Smithsfield, Monroe

Suithstown, Clinton

Smithtown, Woodbury

Snyrna, Clarke

Snapp, Pottawattamic

Snow Hill, Page

Snyder, Dullas

Snyder Grove, Woodbury
Soap Creek, Davis

Soap Creek Mills, Davis

Soaptown, Iowa

Soda Bar, Palo Alto

Soldier Valley, Harrison

Somber, Worth

Somida, Shelby

Sonora, Poweshick

South Augusta, Lee

South̄ Bentonsport, Van Buren

South Flint, Des Moines

South Fork, Wayne

South Franklin, Lee

South Independence, Decatur

South Keosauqua, Van Buren

South Liberty, Johnson

South O'Brien, O'Brien

Southport, Sac

Spencer Grove, Benton

Spencerville, Jones

Spinneyville, Scott

Spring Branch, Delaware

Spring Creek, Tama

Spring Grove, I.inn

Spring Lake, Bremer

Spring Rock, Clinton

Spring Run, Louisa

Spring Valley, Decatur

Spring Valley, Mononi

Springfield, Clayton

Springfield, Jackson

Springfield, Keokuk

Springfield, Muscatine

Springfield, Polk

Springvale, Humboldt

Springville, Clayton

Springville, Davis

Springwater, Winneshick

Spruce Mills, Jackson

Squires Mills, Dubuque

Staketown, Palo Alto

Stanford, Marshall

Stapleton, Chickasiaw

Star, Marion

Steady Run, Keokuk

Steen, Winnebago

Stelapolis, Iowa

Stella, Allamakee 
Stergis, Howard

Sterling, Jackson

Sterling, Louisa

Stilesville, Davis

Stillwater, Mitchell

Stillwell, Poweshiek

Stoneridge, Mahaski

Story, Story

Strand, Adams

Strawberry Hill, Jones

Strawberry Hill, Muscatine

Streepyville, Appanoose

String Prairie, L.ee

Stringtown, Davis

Stringtown, Henry

Stumptown, Van Buren

Sturges Falls, Black Hawk

Sturges Rapids, Blatek Hawk

Suffolk, Clinton

Sugar Creek, Cedar

Sugar Creek, Jasper

Sugar Creek Mills, Cedar

Sugar Grove, Poweshiek

Sullivan, Jackson

Summer Hill, Jackson

Summers, Benton

Summerset, Adair

Summit, Clay

Summit, Story

Summit, Van Buren

Sumner (P. O.), Bremer

Sumner, Humboldt

Sunnyside, Buchanan

Sunset, Story

Sutton, Warren

Swan Lake, Emmet

Swan Lake, Pocahontas

Swanton, Butler

Swea (P. O.), Kossuth

Swede Point, Boone

Sylvan, Clayton

Syracuse, Bremer

'Tallahoma, Lucas

'Talleyrand, Keokuk

Tamaville, Tama

Tanktown, Johuson

Tara, Dubuque

Tarkio, Page
Tarkio City, Page

'Taunton, Warren

Taylor, Davis

'Vaylor Hill, Grundy

Taylor Station, Pottawattamie

'Taylor's Grove, Benton

'Taylorsville, Fayette

'Tecumseh, Louisa

'Temple Hill, Jones

'Tenold, Worth

Tenville, Montgomery

'Terre Haute, Decatur

'Terry, Benton

Tete de Mort, Jackson

'Thomas, Ringgold

'Thompson, Audubon

'Ihompsontown, Woodbury

Thompsonville, Monroe

'Thoten, Winneshiek

Thrall, Wright

'Three Rivers, Polk

'Tibbitt, Polk

'Tibbottsville, Shelby

Tieville, Monona

'Tileville, Madison

Tilton (P. O.), Poweshiek

'T'imber Creek, Marshall

'lime, L.ucas

'lingley (P. O.), Union

lipperary, Iucas

'Iippo, Davis

'lip 'Top, Carroll

Tipton Grove, Hardin

Titus, Washington

'Tivoli, Dubuque

'Toddtown, Louisa

'Tom Corwin, Allamakee

'Tool's Point, Jasper

'Jurall, Limn

'Tower Hill, Delaware

Towner, Polk

'Towner Lake, Polk

'Tracey, Dallas

'Traders Point, Mills

Tranquility, Appanoose

'Traxler, Henry

'Tremaine, Hamilton

Trent, Polk 
'Trimello, Clay

'Tripoli (Old), Bremer

Trout River, Winneshiek

'Troy, Iucas

Tunis, Wapello

'Iunmel, Hamilton

'I'urkey Grove, Cass

'Turkey Run, Decatur

'Turner, Jasper

'Turner, Mills

'Iurnersville, Fayette

'Tuscarora, I.ee

'Tuttle Grove, Guthrie

'Iweten, Winnebago

'Iwelve Mile Grove, Warren

'Twin I.akes, Calhoun

'Twin Oak, Louisa

'Twin Springs (No. 1), Winneshiek

'Twin Springs (No. 2), Winneshiek

'Tyner, Polk'

'Typee, Jefferson

'Tyro, Poweshiek

'Iyson's Mills, Webster

Ulin, Polk

Ulster, Floyd

Una, Wayne

Uncle $\Lambda$ be, Davis

Uncle Sam, Dallas

Union, Polk

Union, Pottawattamie

Union, Van Buren

Union Center, Jackson

Union City, Union

Union Corners, Van Buren

Union Grove, Cedar

Union Grove, Page

Union Hill, Ringgold

Union Hill, Warren

Union Prairie, Allamakee

Union Ridge, Butler

Union Ridge, Franklin

Unnionburg, Harrison

Uniontown, Delaware

Unionville, Boone

Unionville, 'Tama

Unique, Humboldt

Unity, Benton

Unity, Johnson
University Place, Polk

Updegraff, Clayton

Upland, Lyon

Upper Girove, Hancock

Upton, Van Buren

Ulrbana City, Monroe

Ute (P. O.), Monona

Valley, Washington

Valley City, Scott

Valley Farm, l.inn

Valley View, Harrison

Van Buren, Jackson

Van Buren, Lee

Vau Buren, Van Buren

Vandalia, Jasper

Vandenburg, Clinton

Vanderbilt, Linn

Vandyke, Des Moines

Van Nest, Guthrie

Vaughn, Fremont

$V$ egra, Henry

Vega, Jefferson

Venus, Madison

Verbeck, Humboldt

Vernon, Van Buren

Vernon Springs, Howard

Verona, Poweshiek

Vesper, Webster

Vienna, Marshall

Village, Wapello

Village Creek, Allamakee

Vincent, Washington

Vineyard, 'Tama

Vino, Adair

Viola, Delaware

Viola, Dubuque

Viola Center, Audubon

Viona, Humboldt

Virginia Grove, I.ouisa

Viroqua, Jones

$V$ ista, Buchanan

Volga City, Fayette

Vollamova, Clinton

Volney, Allamakee

Von, I Humboldt:

Voss, Allamakee

Vowells, Jasper.

Vulcan, Keokuk 
Wabasha, Clayton

Wacousta, Humboldt

Wadiloup, Grundy

Wagner, Clayton

Wagonershurg, Jackson

Wahaghbonsy, Mills

Wahkonsa, Polk

Wallawwah, Adair

Wakeficld, Cass

Walbridge, Humboldt

Walbridge Grove, Webster

Wald, Cedar

Walden, Keolik

Wales, Montgomery

Wales, Worth

Walkerville, Page

Wallace, Dickinson

Wallace, Montgomery

Walling's Tanding, Louisa.

Walnut, Jefferson

Walnut City, $\Lambda$ ppanoose

Walnut Creek, Fremont

Walnut Creek, Polk

Walnut Creek Station, Pottawattamie

Walnut Fork, Jones

Walnut Grove, Mills

Walnut Grove, Pottawattamie

Walnut Grove, Scott

Walsh, A ppanoose

Waltham, 'Tama

Wanamaker, Ringgold

Waneta, Davis

Wapsa, Iinn

Wapsie, Bremer

Wapsie, Mitchell

Ward, Mills

Ward, Monroe

Ward's Corners, Buchanan

Wardsville, Hancock

Warner's Mill, Keokuk

Warren, Black Hawk

Warren, J.yon

Warren Center, Jucas

Warren Grove, Jasper

Warrensville, Mahaska

Warsaw, Cedar

Warsaw, Wayne
Washburn, Franklin

Washington, Henry

Wassonville, Washington

Waterford, Jackson

Waterman, O'Brien

Waterman, Wright

Watertown, Floyd

Watertown, Van Buren

Watson, Allamakee

Watson, Clayton

Waubesopinecoux, Dubuque

Waudena, Chickasaw

Waugh's Point, Keokuk

Waveland, Pottawattamie

Wax, Cass

Wayne Cross Roads, Wayne

Webster, Allamakec

Webster, Madison

Weeping Willow, Davis

Weld's Landing, Dubuque

Welland, Appanouse

Weller, Monroe

Wells, Madison

Wells Mills, Appanoose

Wellsburg, Page

Welton (Old), Clinton

Wendell, Cherokee

Wentworth, Mitche!l

West Albany, Faycte

West Alexander, Lucas

West Auburn, Fayctte

West Bencl (P. O.), Pialo Alto

West Buffalo, Scott

West Dayton, Webster

West Fork, Monona

West Irving, Tama

West Lake, Hancock

West I.iberty, Polk

West London, Keokuk

West Milton, Guthric

West Pilot, Iowa

West Point, Butler

West Port Louisa, Iouisa

West Prairic, Linn

West Scott, Buena Vista

West Troy, Jowa

West Union, Tamal

West Union, Union 
Western, Linn

Western, Poweshiek

Western City, Keokuk

Western College, I.inn

Westfield, Fayette

Westfield, Poweshiek

Westfield, Woodbury

Westford, Warren

Weston, Marion

Westport, Linn

Wexford, Allamakee

Wheeler, Pottawattamic

Wheeler's Grove, Pottawattamie

Wheeler's Rancl, Sac

Wheeling, Marion

Whipple, Pottawattamie

White, Monroe

White Ash, Washington

White: Breast, Clarke

White Breast (No. 1), I.ucas

White Breast (No. 2), Lucas

White Cloud, Mills

White Elm, Davis

White Iane, I.ee

Wìite Oak, Mahaska

White Oak Point, Warren

White Pigeon, Keokuk

White Sulpher, Scott

Whiteburg, Monroe

Whitesboro, Harrison

Whitefield, Jefferson

Whitneyville, Cass

Wickliffe, Jackson

Widow Post, $\Lambda$ llamakec

Widuw's Home, Mahaski.

Wilkins, Guthrie

Willard, Wapello

Williams, Benton

Williams Point, Wright

Williamsburg, Wapello

Williamstown, Chickasaw

Williamstown, Johnson

Williamsville, Jasper

Willida, I.yon

Willimantic, Winneshick

Willits, Van Buren

Willoughby, Butler

Willow, Pottawattamic
Willow' Creek, Clay

Willow Dale, Ida

Willow Grove, Story

Wilmington, Jienton

Wilmington, Guthrie

Wilmington, Warren

Wilson, Iowa

Wilson, Montgomery

Wilson Grove, Bremer

Wilson's, Guthrie

Wilson's Ford, Allamakee

Wilson's Grove, Butler

Wilson's Grove, Fayctte

Wilsonville, Van Buren

Wimer's Mills, Keokuk

Wincham, Johnson

Windsor, Clayton

Windsor, Fayette

Windsor, Taylor

Winland, Sioux

Winneshiek, Winneshiek

Winoni, Henry

Wirt, Ringgold

Wisconsin, Warren

Wiscotta, Dallas

Wittenburg, Jasper

Wolf Creek, Tama

Wolfdale, Woodbury

Woodbridge, Cedar

Woodbridge, Floyd

Woodbury, Woodbury

Woodlawn, Henry

Woods, Benton

Woods Mills, Van Buren

Woodside, Winneshiek

Woodstock, O'Brien

Woodville, Jasper

Woodville, Winneshiek

Wooleyville, Davis

Woolstock (P. O.), Wright

Wooster, Jefferson

Wooster, Pottuwattamie

Wortl, Boone

Worthington, Madison

Wyoming, Muscatine

Xenia, Dallas

Xenia, Hardin

Yampa, Wapello 
Yankee, Clay

Yankee Grove, Cedar

Yankee Settlement, Delaware

Yankee 'Town, Louisa

Yatesville, Calhoun

Yatton, Washington

Yazoo, Harrison

Yeargerville, Lee

Yellow Banks, Louisa

Yellow Spring, Des Moines

Yeomans, Plymouth

Youngstown, Polk
York, Delaware

York Center, Iowa

York Prairie, Cedar

Yough, Boone

Zanesville, Van Buren

Zarahemla, Lee

Kello, Chickasaw

Zenorsville, Boone

Zero, Lucas

Zoar, Cedar

Zurich, Jones

\section{THE INDIANS ARE FEARED}

The inhabitants of the west side of the Mississippi are complaining loudly of the Indians, who in emigrating west to the district of country allotted them by the government, have squatted down among them. These are the same Indians we alluded to last winter as hunting on government lands. They are at present, as we are informed, extremely insolent, and in many instances have threatened the lives of the settlers. They say that it is their intention to plant corn on the Maquoquita this season, and to drive the whites off from the lands and compel them to take refuge on this side of the Mississippi. The settlers in consequence are alarmed, and in one or two instances have brought their families over to this side. Disagreeable results will shortly happen if there is not something done on the part of the government agents to check the lawless propensities of the Indians and to remove them from the lands. It is said to be hazardous for the settlers to leave their cabins, as the Indians will enter them in their absence, and carry off anything to which they may take a fancy. If there is no other resort the settlers will be obliged to band together for mutual protection and safety.-Western Gazette and Galena Advertiser, Galena, Wisconsin 'I'erritory, April 8, 1837. (In the Newspaper Division of the Historical, Memorial and Art Department of Iowa.) 
Copyright of Annals of Iowa is the property of State of Iowa, by \& through the State Historical Society of Iowa and its content may not be copied or emailed to multiple sites or posted to a listserv without the copyright holder's express written permission. However, users may print, download, or email articles for individual use. 